\title{
Is the Gap in Incidence of Cardiovascular Events in Rheumatoid Arthritis Really Closing?
}

\author{
Joan M. Bathon ${ }^{1}$
}

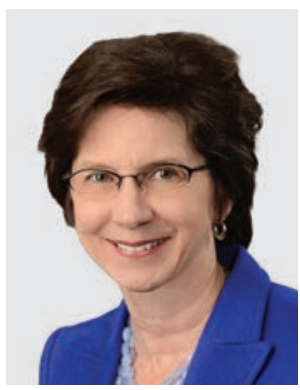

It has been recognized for several decades that individuals with rheumatoid arthritis (RA) have shorter life spans than age- and sex-matched individuals without RA, and that the greatest contribution to this increased mortality in RA is cardiovascular (CV) disease, particularly acute myocardial infarctions (AMIs). In metaanalyses of observational studies, the risk of AMI was $68 \%$ higher, and the risk of mortality from AMI 59\% higher, in patients with RA compared to general population controls. ${ }^{1,2}$ The causal factor hypothesized to explain these CV risk differences is the exaggerated inflammatory process that is inherent to RA and persists throughout the disease course of RA to varying degrees. Atherosclerosis has been demonstrated histologically to be an inflammatory process with infiltration of monocytes and $\mathrm{T}$ cells as an early event in plaque formation. ${ }^{3}$ This intraplaque inflammatory process can lead directly to erosion and rupture of plaque membrane through cytokine-induced metalloproteinase production, leading to extrusion of thrombogenic material into the vessel lumen and consequent thrombosis and ischemic events. ${ }^{3}$ Many of the inflammatory cells and molecules in the inflamed and ruptured plaque are represented in the synovia and blood of patients with RA, suggesting that RA further fuels the atherogenic process. A critical question therefore is whether aggressive reduction of inflammation in RA will reduce the enhanced risk of $\mathrm{CV}$ disease relative to the general population, or even to levels below the general population.

The treatment of RA has changed dramatically in recent decades, beginning with the introduction of methotrexate (MTX) in the late 1980s, anticytokine and other targeted therapies in the late 1990s, and the emphasis on aggressive treat-to-target management of RA in the 2000s. With these

${ }^{1} J . M$. Bathon, MD, Professor of Medicine, Division of Rheumatology, Columbia University Irving Medical Center/New York Presbyterian Hospital, New York, New York, USA.

$J M B$ declares no conflicts of interest relevant to this article.

Address correspondence to Dr. J.M. Bathon, MD, Professor of Medicine,

Division of Rheumatology, Columbia University Irving Medical Center/

New York Presbyterian Hospital, New York, NY 10032, USA.

Email:jmb2311@columbia.edu. advances, there was an expectation that $\mathrm{CV}$ morbidity and mortality in RA would decrease accordingly. Specifically, investigators have asked (1) if CV event and CV mortality rates have declined in RA patient populations since these therapeutic advances became available; and (2) if so, have the gaps in CV event and mortality rates between RA and the general population declined during this time? Indeed, several groups have reported decreased CV mortality rates in patients with RA in the 2000s compared to prior decades. ${ }^{45,6,67}$ However, it is not clear whether this reduced mortality is due to lower numbers of AMIs or due to prolonged survival post-AMI. Moreover, data comparing declining $\mathrm{CV}$ event rates in patients with $\mathrm{RA}$ to rates in the general population over the same periods of time are sparse.

In this issue of The Journal of Rheumatology, Myasoedova, et $a l^{8}$ revisit these questions in an updated investigation to determine whether $\mathrm{CV}$ event rates in patients with RA have declined, and whether the gap in CV event rates between RA and non-RA subjects has narrowed over the last several decades. Using the Rochester Epidemiology Project population-based medical records linkage system, the authors identified Olmsted County, Minnesota residents with incident RA diagnosed during the period of 1980-2009, and non-RA subjects drawn from the same source matched 1:1 to the RA patients for age, sex, and calendar year of index. CV events (AMI and stroke) were identified using standardized diagnostic criteria, and AMIs were adjudicated by a cardiologist. All subjects were followed until death, migration, or the study termination date of December 31, 2016. The final study populations included $905 \mathrm{RA}$ patients with 12,808 person-years (PY) of follow-up, and 904 non-RA subjects with 13,095 PY of follow-up. Subjects were categorized by decade of onset of RA (or index decade for non-RA subjects), specifically 1980-89, 1990-99, and 2000-9.

In the first analysis of CV events (AMI and stroke) in the RA group only, the authors found that, adjusting for age and sex, the risk of any $\mathrm{CV}$ event decreased over time, with a $42 \%$ lower risk in patients diagnosed in the 2000s compared to those diagnosed in the 1980s (HR 0.58, 95\% CI 0.34-0.98). When AMIs

\section{See CVD incidence in RA, page 1379}


were considered separately, a 56\% lower risk was observed (HR $0.44,95 \%$ CI $0.20-0.97$ ) in the 2000 s compared to the 1980 s. In contrast, no significant decrease was observed in stroke rates between decades. The results with any CV event and with AMIs were maintained after adjustment for conventional CV risk factors, indicating that the decline in $\mathrm{CV}$ risk was not simply due to differences in rates of diabetes, hypertension, and the like over time. When the highest erythrocyte sedimentation rate in the first year of disease or disease-modifying antirheumatic drug (DMARD) treatments were put into the models, the difference between the 2000s vs 1980s was attenuated for any CV event, suggesting that the decrease in $\mathrm{CV}$ risk might be related in part to decreased inflammation.

The authors next compared CV event risk between patients with RA and the non-RA controls over the same time segments. In these analyses, the authors found that the HRs for any $\mathrm{CV}$ event in RA vs non-RA subjects were elevated in the first 2 decades of RA incidence (HR 1.66, 95\% CI 1.08-2.56 and HR 2.08 , 95\% CI $1.27-3.40$ for the 1980 s and 1990 s, respectively), whereas there was no excess risk in patients with incident RA in the 2000s (HR 0.71, 95\% CI 0.42-1.19), although there was some attenuation in risk in the 1980s and 1990s cohorts after adjusting for age, sex, and conventional CV risk factors. This observation suggests that the gap in CV risk between RA and the general population has decreased in the modern era, coincident with recent major therapeutic advances in RA. When the authors examined individual events, there were numerical reductions in HRs for AMI and stroke over the decades, but most of the HRs were not statistically significant, possibly due to small sample sizes.

The authors also investigated whether all-cause mortality rates after $\mathrm{CV}$ events differed between $\mathrm{RA}$ and non-RA subjects over the 3 decades of incident disease. All-cause mortality rather than $\mathrm{CV}$-specific mortality rates were examined, presumably due to the small sample sizes. In the RA group, there was a decrease in mortality in the group diagnosed in the 1990s compared to those diagnosed in the 1980 s, but there was no statistically significant decrease in the 2000s group compared to the 1980s group. Moreover, mortality rates in RA vs non-RA patients were not significantly different for any of the 3 decades of disease onset. Given the small total number of mortality events (83 RA, 57 non-RA), this finding could again reflect insufficient power to detect a difference.

This report by Myasoedova, $e t a l^{8}$ adds to the growing data suggesting that $\mathrm{CV}$ event rates-AMIs in particular-are declining in successive incident RA cohorts, but leaves open questions about whether the gaps in $\mathrm{CV}$ event rates and $\mathrm{CV}$ mortality rates between RA and non-RA are truly declining. Other reports of $\mathrm{CV}$ event rates in RA vs non-RA comparators that include time periods after 2000 are scarce. Holmqvist, et al performed a nationwide population-based cohort study in Sweden of 15,744 patients with newly diagnosed RA between 1997 and 2014, and 70,899 non-RA comparator subjects matched on age, birth year, and residential area from the same population and same time period. The incidence rates of new CV events declined by approximately $40 \%$ over the study period for both RA and non-RA subjects. However, patients with RA had a persistently higher incident $\mathrm{CV}$ event rate throughout the time period compared to the non-RA controls (overall HR 1.41, 95\% CI 1.29-1.54). It should be noted, however, that this difference was not statistically significant for the time segment 2010-14, during which the number of $\mathrm{CV}$ events was quite small. Interestingly, whereas Myasoedova, $e t a l^{8}$ reported no difference in incidence rates in rheumatoid factor (RF)-negative vs -positive patients with RA, Holmqvist, et al found that the excess risk of $\mathrm{CV}$ events in patients with RA was driven by RF positivity and higher disease activity at baseline.

More recently, Yazdani, et al ${ }^{\beta}$ reported a retrospective study of the 10-year AMI risk in a population-based cohort of incident RA patients $(\mathrm{n}=23,237)$ in British Columbia, Canada, diagnosed over the period of 1997-2004, compared to matched controls $(n=46,474)$ from the general population over the same time period, using administrative health data. They also reported equivalent rates of decline in risk of AMI in the RA $(0.94,95 \%$ CI $0.91-0.97)$ and non-RA (0.93, 95\% CI 0.91-0.95) groups over the course of the study period, although the crude incidence rates were numerically higher in RA compared to non-RA groups in 7 of the 8 years of study entry. Indeed, Yazdani, et al found a $21 \%$-higher risk of AMI in the RA group compared to the matched general population controls $(1.21,95 \% \mathrm{CI}$ $1.10-1.32$ ) over the course of the study period, after adjusting for potential confounders.

What could be the explanation for the discrepancy in results between the Myasoedova study ${ }^{8}$ showing closing of the CV gap in recent decades, compared to the Yazdani ${ }^{9}$ and Holmqvist ${ }^{7}$ studies, which did not? All were population-based studies with matched comparators, but the Holmqvist ${ }^{7}$ and Yazdani ${ }^{9}$ studies had much larger sample sizes that yielded significantly higher numbers of CV events than Myasoedova, et al. ${ }^{8}$ Thus, the lack of a statistically significant difference in $\mathrm{CV}$ event rates between the RA and non-RA groups in the 2000s vs the 1980s in the Myasoedova study ${ }^{8}$ may have been due to inadequate power. A strength of the Myasoedova study was that all AMIs were adjudicated by a cardiologist, whereas the other 2 studies utilized International Classification of Diseases codes for AMI diagnosis, although the latter 2 studies reported excellent positive predictive values for this methodology. ${ }^{7,9}$ Another particular strength of the Myasoedova study was the availability of robust medical records that yielded detailed information on conventional CV risk factors and allowed for statistical adjustment for these potential confounders in the RA vs non-RA event comparisons. ${ }^{8}$ In contrast, Holmqvist, et al ${ }^{7}$ did not report on these confounders, and Yazdani, et al ${ }^{9}$ presentation of them was arguably less robust than Myasoedova, et al. ${ }^{8}$ However, despite Myasoedova, et als rich data resource, RA disease activity measures were apparently not systematically gathered; they were also not available in the administrative dataset examined in the Yazdani study. ${ }^{8,9}$ In contrast, Holmqvist, et al had robust measures of RA disease activity, enabling the authors to investigate and report on the significant contribution of disease activity to the risk of subsequent $\mathrm{AMI}$ in the RA group. 
In summary, while data are consistent and encouraging in identifying a substantial decrease in AMI rates in newly diagnosed patients with RA over consecutive decades, it is still unclear whether the gap in AMI rates between RA and the general population has truly narrowed or not. Additional studies that extend through the current decade may be useful to answer this question as they would have the advantage of a longer period of exposure to aggressive treatment of RA. However, this will likely be counterbalanced by the diminishing absolute number of events occurring in both RA and non-RA patients, which may continue to obscure differences in $\mathrm{CV}$ event rates between groups, if they exist. Meanwhile, current work must continue to focus on understanding what feature(s) of RA drives the increased CV risk, how conventional CV risk factors interact with RA-specific features to enhance risk, and whether and which RA DMARDs reduce and/or enhance risk. For now, it is comforting to know that $\mathrm{CV}$ events in patients with $\mathrm{RA}$ are at least decreasing at rates equivalent to the general population.

\section{REFERENCES}

1. Avina-Zubieta JA, Thomas J, Sadatsafavi M, Lehman AJ, Lacaille D. Risk of incident cardiovascular events in patients with rheumatoid arthritis: a meta-analysis of observational studies. Ann Rheum Dis 2012;71:1524-9.

2. Avina-Zubieta JA, Choi HK, Sadatsafavi M, Etminan M, Esdaile JM, Lacaille D. Risk of cardiovascular mortality in patients with rheumatoid arthritis: a meta-analysis of observational studies. Arthritis Care Res 2008;59:1690-7.
3. Libby P. Inflammation in atherosclerosis. Arterioscler Thromb Vasc Biol 2012;32:2045-51.

4. Lacaille D, Avina-Zubieta JA, Sayre EC, Abrahamowicz M. Improvement in 5-year mortality in incident rheumatoid arthritis compared with the general population-closing the mortality gap. Ann Rheum Dis 2017;76:1057-63.

5. GBD 2015 Mortality and Causes of Death Collaborators. Global, regional, and national life expectancy, all-cause mortality, and cause-specific mortality for 249 causes of death, 1980-2015: a systematic analysis for the Global Burden of Disease Study 2015. Lancet 2016;388:1459-544.

6. Myasoedova E, Gabriel SE, Matteson EL, Davis JM 3rd, Therneau TM, Crowson CS. Decreased cardiovascular mortality in patients with incident rheumatoid arthritis (RA) in recent years: dawn of a new era in cardiovascular disease in RA? J Rheumatol 2017; 44:732-9.

7. Holmqvist M, Ljung L, Askling J. Mortality following new-onset rheumatoid arthritis: has modern rheumatology had an impact? Ann Rheum Dis 2018;77:85-91.

8. Myasoedova E, Davis JM 3rd, Roger VL, Achenbach SJ, Crowson CS. Improved incidence of cardiovascular disease in patients with incident rheumatoid arthritis in the 2000s: a population-based cohort study. J Rheumatol 2021;48:1379-87.

9. Yazdani K, Xie H, Avina-Zubieta JA, Zheng Y, Abrahamowicz M, Lacaille D. Has the excess risk of acute myocardial infarction in rheumatoid arthritis relative to the general population declined? A population study of trends over time. Semin Arthritis Rheum 2021 2;51:442-9. 